\title{
Analysis of CPW-Fed Back-to-Back Patch Antenna on Finite-Size Substrate
}

\author{
I-Jen Chen and Powen Hsu
}

Department of Electrical Engineering, Rm. 544

National Taiwan University, Taipei, Taiwan 10617, R.O.C

Tel: +886-2-23635251 ext 544; Email: phsu@cc.ee.ntu.edu.tw

\begin{abstract}
A CPW-fed back-to-back patch antenna printed on a finite-size substrate is analyzed by using the FDTD method. The variations of input impedance and resonance frequency with the widths of the rectangular patch and the substrate are calculated and compared to the existing experimental data. Good agreement between theory and experiment is obtained and a strong finite-size effect is observed.
\end{abstract}

\section{Introduction}

It has been shown recently that the CPW-fed back-to-back patch antenna [1] or large slot-loop coupled patch antenna [2] can radiate an omnidirectional pattern in the H-plane, and thus is suitable for wireless applications if the substrate width is cut to be as narrow as possible. However, the results presented in both [1] and [2] are obtained mainly from experiments. For design purpose, it is necessary that an analysis method be developed to calculate the effect of the finite-size substrate on the characteristics of the antenna. In this paper, an FDTD method is employed to calculate the input impedance and resonant frequency of the CPW-fed back-to-back patch antenna fabricated on a narrow substrate. The results are compared to the experimental data presented in [1]. Good agreement is obtained.

\section{FDTD Modeling of the Antenna}

The configuration of a CPW-fed back-to-back rectangular patch antenna is shown in Fig. 1. Two identical rectangular patches are used and each patch is arranged back-to-back relative to the CPW on the ground plane. Since there are many references that can be found in the open literature regarding to the FDTD method, we describe here only the basic assumptions and parameters used in our formulation of the problem. A standard procedure described in [3] is used to obtain the frequency dependent characteristics of the antenna. As depicted in Fig. 2, a Gaussian pulse is excited in a CPW line at the source plane of the computation domain. The voltage and current are recorded at the reference plane by integrating the electric and magnetic fields. 
The substrate parameters used in the simulation are $\epsilon_{\mathrm{r}}=2.6$ and $t=$ $1.6 \mathrm{~mm}$. At the air-dielectric interfaces, the average dielectric constants are used, i.e., $\left(\epsilon_{r}+1\right) / 2$ at the plane interfaces and $\left(\epsilon_{r}+3\right) / 4$ at the edges. The metal conductors are assumed to be perfect conductors with zero thickness and modeled by setting the tangential electric field components on this plane to zero. The dimensions of the FDTD unit cell, $\Delta x, \Delta y$, and $\Delta z$, are chosen equally as $0.4 \mathrm{~mm}$. To make the integral numbers of nodes fit exactly all the dimensions of the circuit, the length $L_{p}$ and the width $W_{p}$ of the rectangular patch were determined at a resonance frequency of $1.9 \mathrm{GHz}$. Therefore, $L_{p}$ is $47.2 \mathrm{~mm}$ and the length of the substrate $L_{s}$ is $150 \mathrm{~mm}$. The widths of the CPW are $W_{o}=6.8 \mathrm{~mm}$ and $W_{i}=4.4 \mathrm{~mm}$, which correspond to a characteristic impedance of $65 \Omega$. The widths of the center strip and slot of the CPW are equal to $11 \Delta x$ and $3 \Delta x$, respectively.

The $65 \Omega$ line was chosen because of the difficulty in modeling a $50 \Omega$ line. A $50 \Omega$ line requires a small CPW slot so that $\Delta x$ is small and the resulting computational domain becomes too large for our computer to handle. The Berenger's perfectly matched layer (PML) absorbing boundary condition [4] was applied to all outer boundaries. As a check on our computer program, published results for the impedance of a microstrip-fed patch antenna [3] were successfully reproduced. The open end CPW gap is placed at the center of the patches and the width is chosen as $1.6 \mathrm{~mm}$.

\section{Results and Discussions}

Fig. 3 shows the simulated real and imaginary parts of the input impedance with the width $W_{s}$ of the substrate fixed at $50 \mathrm{~mm}$ and the width $W_{p}$ of the rectangular patch changed from 50 to $20.4 \mathrm{~mm}$. The resonance frequencies can be read from the figures at the point where the imaginary part of the input impedance is zero. From the simulated results, the following observation is clear: by decreasing the $\frac{W_{p}}{W}$ ratio, the resonance frequency and the input resistance are both increased. Table 1 summarizes the simulated input impedances and resonance frequencies as parameters of $W_{s}$ and $W_{p}$. These data were compared to those listed in Table 2, which were measured by the experiments in [1], with similar variations.

\section{Conclusion}

The effect of the finite-size substrate on the input characteristics of the CPW-fed back-to-back patch antenna has been calculated by using the FDTD method. The results are compared to the existing experimental data with good agreement. However, ther are still some difficulties to overcome. As the antenna element becomes larger or the CPW slot becomes smaller, the present analysis method will become computationally difficult to model the problem. Thus, a more general and computational saving numerical technique still needs to be developed to handle this problem. 


\section{REFERENCES}

[1] H. Iwasaki, "A back-to-back rectangular-patch antenna fed by a CPW," IEEE Trans. Antennas and Propagat., vol. 46, pp. 1527-1530, Oct. 1998.

[2] S. W. Lu, T. F. Huang, and P. Hsu, "CPW-fed slot-loop coupled patch antenna on narrow substrate," Electron. Lett., vol. 35, no. 9, pp. 682-683, Apr. 1999.

[3] D. M. Sheen, S. M. Ali, M. D. A bouzahra, and J. A. Kong, "Application of the three-dimensional finite difference time domain method to the analysis of planar microstrip circuit," IEEE Trans. Microwave Theory and Tech., vol. 38 , pp. $849-857$, July 1990

[4] J.-P. Berenger, "A perfectly matched layer for the absorption of electromagnetic waves," J. Comput. Phys., vol. 114, no. 2, pp. 185-200, Oct. 1994.

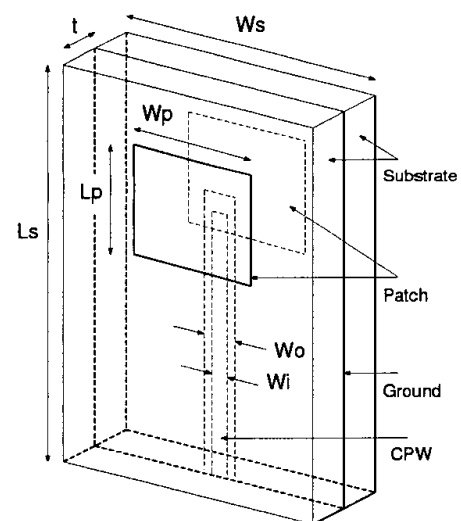

Fig. 1. Configuration.

TABLE 1

Summarized Simulational Results

\begin{tabular}{|c|c|c|c|c|}
\hline $\begin{array}{r}W_{s} \\
W_{p}(\mathrm{~mm})\end{array}$ & 20.4 & 25.2 & 50.0 & 70.0 \\
\hline \multirow{2}{*}{46.8} & \multicolumn{2}{|l|}{ Resonant Freq. } & $1.87 \mathrm{G}$ & $1.89 \mathrm{G}$ \\
& \multicolumn{2}{|l|}{ Input Resist. } & $43.2 \Omega$ & $58.1 \Omega$ \\
\hline \multirow{2}{*}{30.0} & & & $1.92 \mathrm{G}$ & $1.92 \mathrm{G}$ \\
& & & $123.6 \Omega$ & $139.5 \Omega$ \\
\hline \multirow{2}{*}{25.2} & & $1.91 \mathrm{G}$ & $1.93 \mathrm{G}$ & $1.93 \mathrm{G}$ \\
& & $93.6 \Omega$ & $165.4 \Omega$ & $182.3 \Omega$ \\
\hline \multirow{2}{*}{20.4} & $1.92 \mathrm{G}$ & $1.93 \mathrm{G}$ & $1.95 \mathrm{G}$ & $1.95 \mathrm{G}$ \\
& $125.7 \Omega$ & $157.6 \Omega$ & $251.5 \Omega$ & $273.7 \Omega$ \\
\hline
\end{tabular}

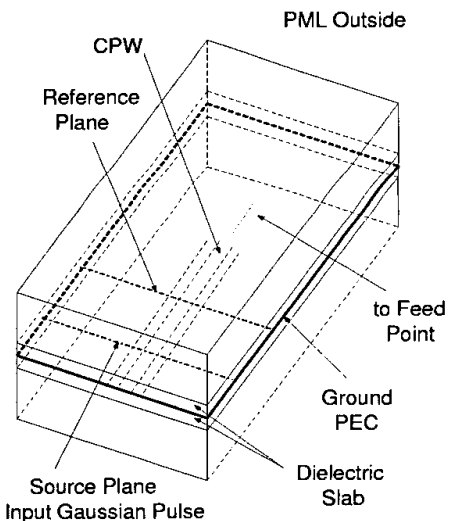

Fig. 2. Excitation

TABLE 2

Summarized Experimental Results

\begin{tabular}{|c|c|c|c|c|}
\hline $\begin{array}{r}W_{s} \\
W_{p}(\mathrm{~mm})\end{array}$ & 20.0 & 25.0 & 50.0 & 70.0 \\
\hline \multirow{2}{*}{47.0} & \multicolumn{2}{|c|}{ Resonant Freq. } \\
& Input Resist. & $1.87 \mathrm{G}$ & $1.89 \mathrm{G}$ \\
\hline \multirow{2}{*}{30.0} & & & $1.89 \mathrm{G}$ & $56.7 \Omega$ \\
& & & $58.9 \Omega$ & $90.5 \Omega$ \\
\hline \multirow{2}{*}{25.0} & & $1.89 \mathrm{G}$ & $1.90 \mathrm{G}$ & $1.92 \mathrm{G}$ \\
& & $63.6 \Omega$ & $78.8 \Omega$ & $112.1 \Omega$ \\
\hline \multirow{2}{*}{20.0} & $1.91 \mathrm{G}$ & $1.92 \mathrm{G}$ & $1.94 \mathrm{G}$ & $1.95 \mathrm{G}$ \\
& $73.9 \Omega$ & $97.8 \Omega$ & $148.8 \Omega$ & $182.5 \Omega$ \\
\hline
\end{tabular}



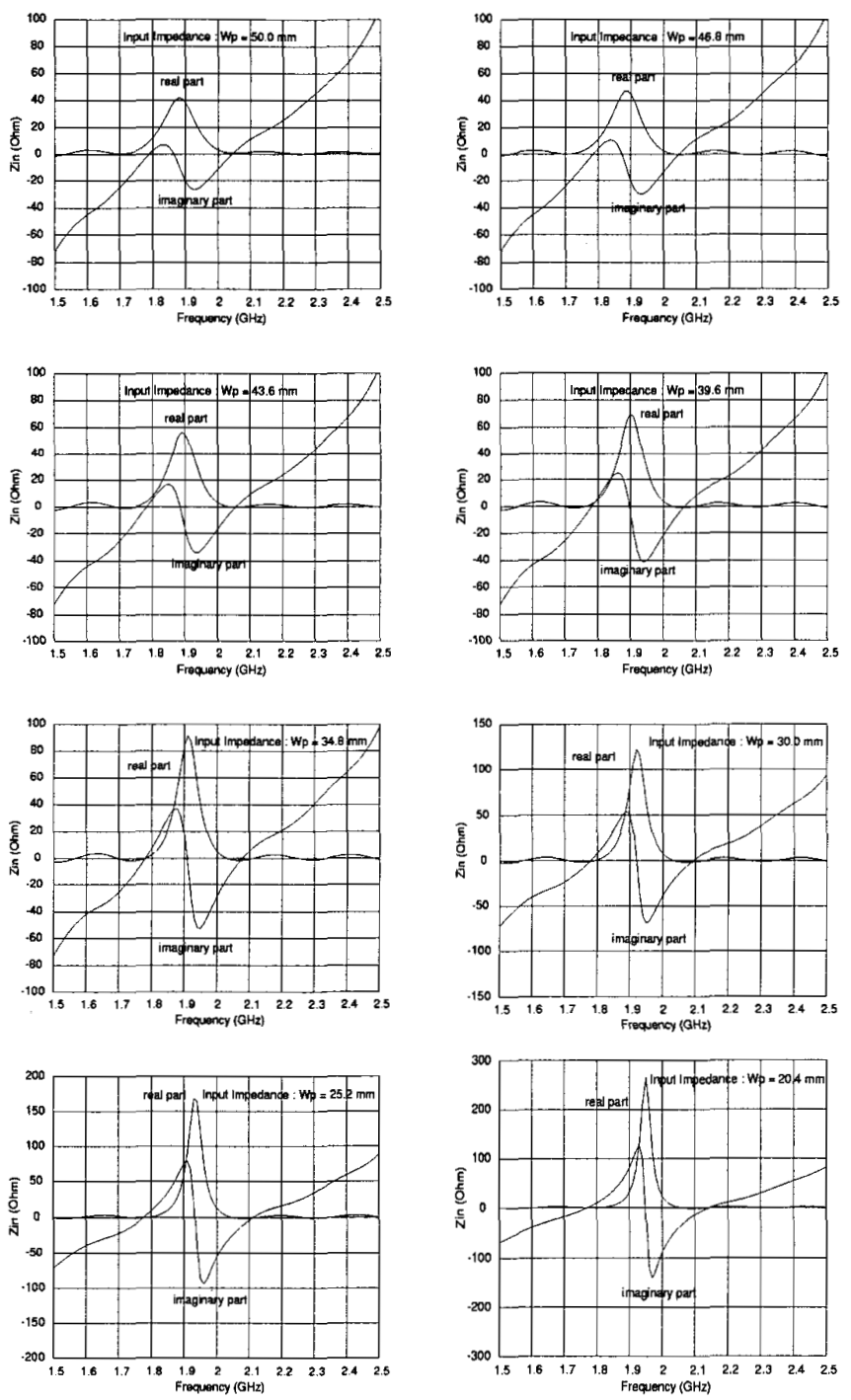

Fig. 3. Input impedance of back-to-back patch antenna. $W_{s}=50 \mathrm{~mm}$. 